\title{
Pulse Pedestal Suppression Using Four-Wave Mixing in an SOA
}

\author{
B. F. Kennedy, K. Bondarczuk, D. A. Reid, and L. P. Barry
}

\begin{abstract}
Experimental results are presented demonstrating how four-wave mixing in a semiconductor optical amplifier can be used to remove pulse pedestals introduced due to nonlinearities which occur upon pulse propagation in an optical system. Such pedestals would degrade the performance of an optical time-division-multiplexed system due to coherent interaction between channels. An improvement of the temporal pulse suppression ratio to greater than $30 \mathrm{~dB}$ is achieved regardless of the level of the pulse pedestal on the input signal. This improvement takes place simultaneously with wavelength conversion and compression of the optical pulse.
\end{abstract}

Index Terms-Optical pulse shaping, optical signal processing, semiconductor optical amplifiers (SOAs).

\section{INTRODUCTION}

$\mathbf{M}$ $\mathrm{UCH}$ research has focused on the implementation of optical time-division multiplexing (OTDM) as a means to realize future high-speed optical communications systems [1]. A vital component of any such system is the picosecond pulse used to carry the optical data. One aspect of such pulses which requires particular attention for OTDM systems is the temporal pedestal suppression ratio (TPSR) of the pulses. For example, it has been reported that a TPSR of $30 \mathrm{~dB}$ is required for the successful operation of a 40-Gb/s OTDM system [2]. This level is difficult to maintain using the typical picosecond pulse generation techniques of mode-locking, gain-switching, and using electroabsorption modulators (EAMs). For these reasons, several techniques have been put forward to improve the TPSR to levels required for error-free operation of OTDM systems. Such techniques include those which use an EAM [3], a nonlinear amplifying loop mirror (NALM) [4], self-phase modulation in a semiconductor optical amplifier (SOA) [5] and, recently, a micro-cavity-based saturable absorber [6].

However, even if a TPSR greater than $30 \mathrm{~dB}$ is achieved using the techniques described above the TPSR also degrades as the pulses propagate through the system due to nonlinearities in the transmission fiber [7], and due to gain saturation effects in SOAs [8]. These devices are widely seen as key

Manuscript received September 14, 2007; revised November 13, 2007. This work was supported by Fondo Nacional de Desarrollo Científico y Tecnológico (FONDECYT) under a research project.

B. F. Kennedy is with the Electrical Engineering Department, Universidad de Santiago de Chile, Estacion Central, Santiago, Chile (e-mail: bkennedy@ lauca. usach.cl).

K. Bondarczuk, D. A. Reid, and L. P. Barry are with the RINCE, School of Electronic Engineering, Dublin City University, Dublin 9, Ireland (e-mail: liam.barry@dcu.ie).

Color versions of one or more of the figures in this letter are available online at http://ieeexplore.ieee.org.

Digital Object Identifier 10.1109/LPT.2007.915643 components in future optical systems [9]. In this letter, a novel technique is put forward to restore the TPSR to greater than $30 \mathrm{~dB}$, while simultaneously wavelength-converting the signal and compressing the four-wave-mixing (FWM) signal pulsewidth. This technique uses the gating properties of FWM in a multiquantum-well SOA. In this process, optical beating occurs between a pump and a probe signal to generate a conjugate signal whose wavelength is determined by the wavelength detuning between the pump and probe signal. Results have been presented in the literature demonstrating the regeneration properties of FWM in SOAs at data rates of $2.5 \mathrm{~Gb} / \mathrm{s}$, but not for the specific case of pedestal suppression [10], [11]. Furthermore, no experimental results have been presented for pulses as short as 2 ps. No experimental results have been presented which completely characterize the pulse waveforms of input pump-probe and output FWM signals. For pulse pedestal suppression, the signal with low TPSR acts as the probe signal. An FWM signal is only generated when the pump and probe intensities are large enough, therefore, the probe pedestal is effectively removed in the FWM process as the pump power is negligible at this position. As well as the suppression of the TPSR, the input signal is also wavelength-converted by the FWM process. Furthermore, the FWM signal has a narrower pulsewidth than the injected probe due to the operation of the FWM process [12], [13]. This multifunction property of the technique put forward is advantageous as it would limit the complexity, and therefore cost, of the overall optical system.

\section{EXPERIMENTAL SETUP}

The experimental setup is illustrated in Fig. 1. Two actively mode-locked laser sources were used to produce optical pulses with duration around 2 ps. The repetition rate of both signals was $10 \mathrm{GHz}$ and the wavelength of the pump laser was $1555 \mathrm{~nm}$ for each measurement taken. This wavelength is close to the peak gain wavelength of the SOA under test. The wavelength of the probe laser was fixed at $1565 \mathrm{~nm}$. The probe signal was amplified and a bandpass filter (BPF) with a bandwidth of $2 \mathrm{~nm}$ was used in order to remove amplified spontaneous emission (ASE) introduced by the erbium-doped fiber amplifier (EDFA). The pulse pedestal on the probe signal was introduced in the same manner as in [6]. A 50:50 coupler was used to split the probe signal. One arm was used as the main probe signal, while the pulse pedestal was introduced using the other arm. An optical delay line (ODL) was inserted to vary the temporal position of the pedestal with respect to the main probe pulse. Variable optical attenuators (VOAs) were placed in both arms of the coupler in order to control the intensity of both signals. It was also necessary to place a polarization controller (PC) 


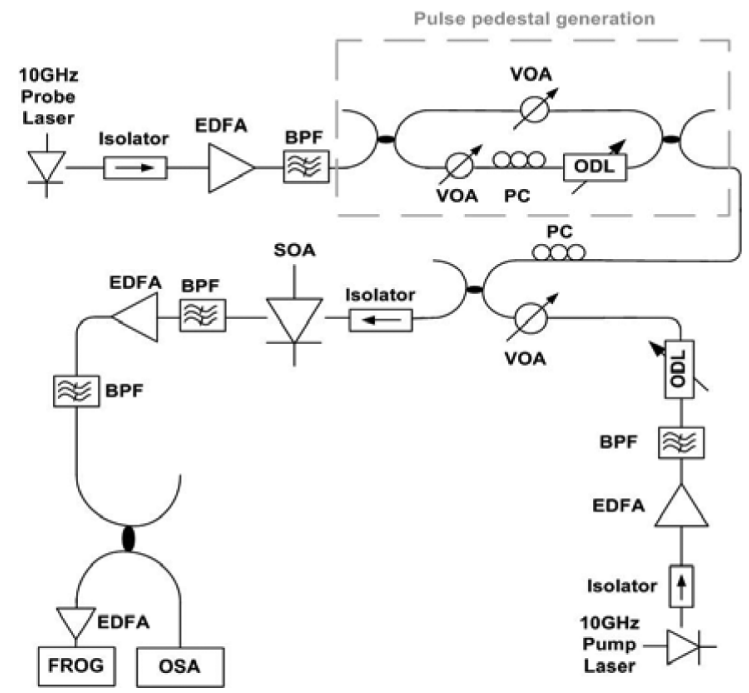

Fig. 1. Experimental setup used to perform FWM in the SOA under test.

in the pedestal arm of the coupler in order to ensure that this signal was copolarized with the other signals before injection into the polarization-sensitive frequency resolved optical gating (FROG) measurement system. The recombined probe signal was then coupled with the pump signal before injection into the SOA. The pump signal was amplified and filtered by a BPF with a passband of $5 \mathrm{~nm}$. The duration of the pump pulse injected into the SOA was $2.32 \mathrm{ps}$, and that of the probe pulse was $2.65 \mathrm{ps}$. The optimum average powers of the pump and probe signals were 4 and $-10 \mathrm{dBm}$, respectively. These powers were found by maximizing both the conversion efficiency and signal-to-background ratio (SBR) using the FWM spectra measured with an optical spectrum analyzer (OSA) of resolution $0.07 \mathrm{~nm}$. A VOA was placed in the pump arm to optimize its power.

An ODL was also placed in the pump arm of the setup to allow for optimum overlapping of the two signals, to produce the most efficient FWM signal.

The SOA under test was a multiquantum-well device biased at $120 \mathrm{~mA}$, with a peak fiber-to-fiber gain of $25 \mathrm{~dB}$. The length of the active region of the device was $600 \mu \mathrm{m}$ followed by linearly tapered active regions of $500 \mu \mathrm{m}$ at each facet [14]. At the output of the SOA, BPFs were used to isolate the FWM component from the output spectrum and to remove ASE introduced by the EDFA. The bandwidth of both BPFs was $5 \mathrm{~nm}$. The FWM component was then examined using second-harmonic generation FROG [15]. The FROG technique generates a three-dimensional spectrogram, which is a time-frequency representation of the pulse. A phase retrieval program is then applied to allow for the electric field of the pulse to be determined, giving complete temporal and spectral characterization of the pulse [16]. Due to the power requirements of the FROG measurement, an EDFA is used to amplify the pulses before they are input into the FROG measurement system. Low errors of $\approx 4 \times 10^{-5}$ were recorded between the data measured experimentally and those obtained using the retrieval algorithm, which indicates high accuracy [15].

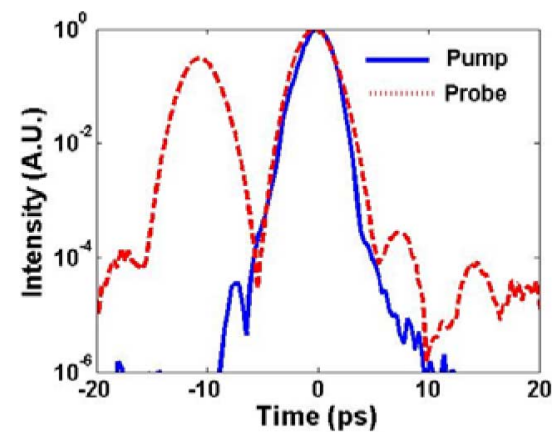

Fig. 2. Temporal alignment of the probe signal with respect to the pump signal before injection into the SOA.

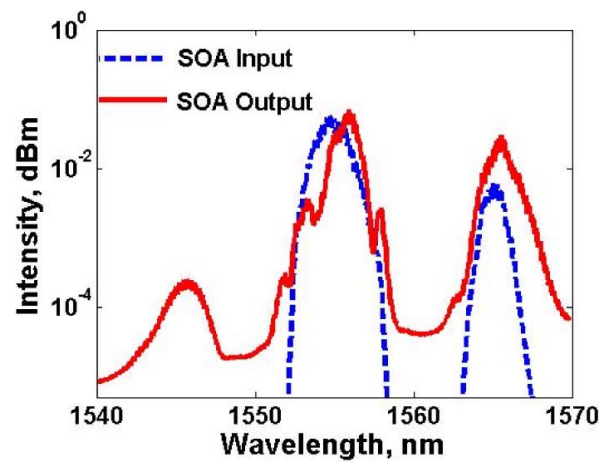

Fig. 3. SOA input and output spectra for a $10-\mathrm{nm}$ detuning.

\section{RESULTS AND DISCUSSION}

The pulse waveforms of the injected pump and probe signals, measured using the FROG system are shown in Fig. 2. The temporal overlap between the pump and probe signals can be seen in this figure. The pump signal has a large TPSR of over $40 \mathrm{~dB}$. However, it should be noted that the error floor of the FROG is $30 \mathrm{~dB}$; therefore, it can be said with confidence that the TPSR is at least $30 \mathrm{~dB}$. Once the TPSR of the pump signal is at least $20 \mathrm{~dB}$, the TPSR of the FWM signal would be large as there would not be sufficient optical power in the beating between the pump pedestal and the probe pedestal to cause significant FWM. The pedestal of the probe signal is located $7 \mathrm{~dB}$ below the peak of the probe, with a temporal delay of approximately $11 \mathrm{ps}$. This value was chosen as it is close to the position where the pedestal would have the greatest impact in a 100-Gb/s system.

The optical spectra of the injected pump and probe signals together with those of the signals at the output of the SOA are shown in Fig. 3. The efficiency of the FWM component is $-12.5 \mathrm{~dB}$ and the SBR is $9.5 \mathrm{~dB}$, indicating a strong FWM component. The FWM component is generated at a wavelength of around $1545 \mathrm{~nm}$.

In Fig. 4, the FWM signal generated due to the beating between the pump and a probe signal is presented, together with the initial probe signal. The TPSR of the conjugate signal is $40 \mathrm{~dB}$; however, as already stated, the FROG has an error floor of approximately $30 \mathrm{~dB}$. Therefore, we can say with full confidence that the improvement in the TPSR is at least $23 \mathrm{~dB}$. The removal of the probe pedestal is due to the absence of any beating between this pedestal and the pump signal. For 


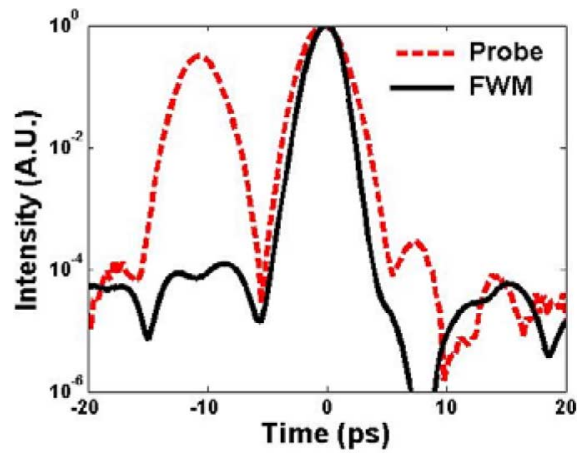

Fig. 4. Probe signal injected into the SOA and FWM signal generated due to the beating between the pump and the probe.

this reason, the pedestal is effectively removed by the FWM process and the only limitation to the OSNR of the FWM signal is caused by the ASE noise from the SOA, on which the FWM signal is sitting, and the relatively low efficiency of the FWM process. It is important to state that the pump pulsewidth is a critical parameter in achieving a TPSR above $30 \mathrm{~dB}$ by this technique. For example, results have been presented which show a TPSR of below $20 \mathrm{~dB}$ when the pump pulse was broadened to 4 ps [17]. Another feature of the generated FWM signal is the pulse compression with respect to the injected probe signal. The pulsewidth of the initial probe signal is $2.65 \mathrm{ps}$, while the FWM signal has a pulsewidth of $2 \mathrm{ps}$. This compression is expected as the FWM signal is proportional to $A_{0}^{2} \cdot A_{1}$, where $A_{0}$ and $A_{1}$ represent the envelopes of the pump and probe signals, respectively [12], [13].

The TPSR of the injected probe signal was then varied using the VOA in the pedestal arm of the probe coupler, shown in the experimental setup in Fig. 1. The TPSR was varied from 7 to $27 \mathrm{~dB}$ and the TPSR of the FWM signal was measured. Regardless of the input probe TPSR, the FWM signal TPSR is consistently greater than $30 \mathrm{~dB}$. This is an important result as the TPSR of the probe signal may vary in a real system. It should also be noted that no degradation of the FWM efficiency or SBR was measured as the intensity of the probe pedestal was varied.

\section{CONCLUSION}

A novel technique has been presented to improve the TPSR of 2-ps pulses, at a repetition rate of $10 \mathrm{GHz}$ using FWM in a multiquantum-well SOA. The main section of the probe signal is gated with a high intensity pump signal in the FWM process. The input TPSR of the probe signal was varied from 7 to $27 \mathrm{~dB}$ and an FWM TPSR of above $30 \mathrm{~dB}$ was consistently measured. The efficiency of the FWM signal was $-12.5 \mathrm{~dB}$ and the SBR was $9.5 \mathrm{~dB}$. Simultaneous wavelength conversion of the probe signal is achieved from 1565 to $1545 \mathrm{~nm}$ and the FWM process compresses the signal from 2.65 to 2 ps.

\section{REFERENCES}

[1] M. J. O'Mahony, "Optical multiplexing in fiber networks: Progress in WDM and OTDM," IEEE Commun. Mag., vol. 33, no. 12, pp. 82-88, Dec. 1995.

[2] P. L. Mason, A. Wonfor, D. D. Marcenac, D. G. Moodie, M. C. Brierley, R. V. Penty, I. H. White, and S. Bouchale, "The effects of pedestal suppression on gain-switched laser sources for $40 \mathrm{Gbit} / \mathrm{s}$ OTDM transmission," in Proc. IEEE Lasers and Electro-Optics Society Annual Meeting, Nov. 1997, vol. 1, pp. 289-290, Paper TuS2.

[3] P. Gunning, J. K. Lucek, D. G. Moodie, K. Smith, R. P. Davey, S. V. Chernikov, M. J. Guy, J. R. Taylor, and A. S. Siddiqui, "Gainswitched DFB laser diode pulse source using continous wave light injection Forjitter suppression and an electroabsorption modulator for pedestal suppression," Electron. Lett., vol. 32, pp. 1010-1011, 1996.

[4] M. C. Gross, M. Hanna, K. M. Patel, and S. E. Ralph, "Reduction of power fluctuations in ultrafast optically time-division-multpilexed pulse trains by use of a nonlinear amplifying loop mirror," IEEE Photon. Technol. Lett., vol. 14, no. 5, pp. 690-692, May 2002.

[5] Z. Hu, M. Davanco, and D. J. Blumenthal, "Extinction ratio improvement by strong external injection and SPM in an SOA for OTDM pulse using a DBR laser diode," IEEE Photon. Technol. Lett., vol. 15, no. 10, pp. 1419-1421, Oct. 2003.

[6] A. M. Clarke, P. M. Anandarajah, L. Bramerie, C. Guignard, R. Maher, D. Massoubre, A. Shen, J. L. Oudar, L. P. Barry, and J. C. Simon, "80-Gb/s OTDM system analysis of a vertical microcavity-based saturable absorber for the enhancement of pulse pedestal suppression," IEEE Photon. Technol. Lett., vol. 19, no. 5, pp. 321-323, Mar. 1, 2007.

[7] M. Nakazawa, "Solitons for breaking barriers to terabit/second WDM and OTDM transmission in the next millennium," IEEE J. Sel. Topics Quantum Electron., vol. 6, no. 6, pp. 1332-1343, Nov./Dec. 2000.

[8] A. M. Clarke, M. J. Connelly, P. Anandarajah, L. P. Barry, and D. A. Reid, "Investigation of pulse pedestal and dynamic chirp formation on picosecond pulses after propagation through an SOA," IEEE Photon. Technol. Lett., vol. 17, no. 9, pp. 1800-1802, Sep. 2005.

[9] J. Mørk, M. L. Nielsen, and T. W. Berg, "The dynamics of semiconductor optical amplifiers: Modeling and applications," Opt. Photon. News, pp. 42-48, Jul. 2003.

[10] H. Simos, A. Argyris, D. Kanakidis, E. Roditi, A. Ikiades, and D. Syvridis, "Regenerative properties of wavelength converters based on FWM in a semiconductor optical amplifier," IEEE Photon. Technol. Lett., vol. 15, no. 4, pp. 556-559, Apr. 2003.

[11] C. Gosset and G.-H. Duan, "Extinction ratio improvement and wavelength conversion based on four-wave mixing in a semiconductor optical amplifier," IEEE Photon. Technol. Lett., vol. 13, no. 2, pp. 139-141, Feb. 2001

[12] N. K. Das, T. Kawazoe, Y. Yamayoshi, and H. Kawaguchi, "Analysis of optical phase-conjugate characteristics of picosecond four-wave mixing signals in semiconductor optical amplifiers," IEEE J. Quantum Electron., vol. 37, no. 1, pp. 55-62, Jan. 2001.

[13] J. M. Tang and K. A. Shore, "Characteristics of optical phase conjugation of picosecond pulses in semiconductor optical amplifiers," IEEE J. Quantum Electron., vol. 35, no. 7, pp. 1032-1040, Jul. 1999.

[14] A. E. Kelly, I. F. Lealman, L. J. Rivers, S. D. Perrin, and M. Silver, "Polarisation insensitive, $25 \mathrm{~dB}$ gain semiconductor laser amplifier without antireflection coatings," Electron. Lett., vol. 32, no. 19, pp. 1835-1836, Sep. 1996.

[15] R. Trebino, K. W. Long, D. N. Fittinghoff, J. N. Sweetser, M. A. Krumbugel, and B. A. Richman, "Measuring ultrashort laser pulses in the time-frequency domain using frequency resolved optical gating," Rev. Sci. Instrum., vol. 68, pp. 3277-3295, 1997.

[16] D. J. Kane, "Real-time measurement of ultrashort laser pulses using principal component generalized projections," IEEE J. Sel. Topics Quantum Electron., vol. 4, no. 2, pp. 278-284, Mar./Apr. 1998.

[17] B. F. Kennedy, K. Bondarczuk, and L. P. Barry, "Detuning dependence of four-wave mixing between picosecond pulses in a multi-quantum well semiconductor optical amplifier," in IMOC 2007, Salvador, Brazil, Nov. 2007. 